\title{
COMPARACIÓN DIALECTAL DEL ESPAÑOL LIMEÑO Y MADRILEÑO: CONTRASTE ACÚSTICO Y PERCEPTIVO DE LAS VOCALES
}

CROSS-DIALECTAL COMPARISON OF LIMA AND MADRID SPANISH: ACOUSTIC AND PERCEPTUAL COMPARISON OF THE VOWELS 
En el presente estudio, se plantea comprobar que existen diferencias acústicas entre los sistemas vocálicos del español madrileño y del español limeño. A partir de esta propuesta, se tiene como objetivo evidenciar que las vocales del español limeño registran mayor duración que las del español madrileño, y que existen diferencias temporales en la percepción de las vocales entre ambas variedades del español. Con el fin de comprobar lo propuesto, se realizaron dos pruebas (una de percepción y otra de producción) que fueron presentadas a 40 participantes (20 madrileños y 20 limeños) con edades entre los 19 y 25 años. Los resultados de la investigación muestran que las vocales de los hablantes de español limeño presentan mayor duración que las de los hablantes de español madrileño, duración que, además, es percibida y categorizada como propia de su variedad dialectal. En cambio, a pesar de que los hablantes de español madrileño producen las vocales con menor duración, esta no es percibida.

PALABRAS CLAVE: comparación dialectal, variedades del español, vocales, indicio temporal

In this study, we suggest to verify if there are acoustic differences on Madrid and Lima Spanish vocalic systems. From this suggestion, we recommend to evidence that Lima Spanish vowels register more duration than Madrid vowels, and that there exist temporary differences in vowels perception between both Spanish varieties. In order to verify this, two tests (perception test and production test) were applied to 40 participants (20 from Madrid and 20 from Lima) from 19 to 25 years old. As a result, the investigation shows that vowels from Lima Spanish speakers demonstrate more duration than Madrid Spanish speakers, duration that, besides, is perceived and categorized as its own dialectal variety. However, even though Madrid Spanish speakers produce vowels with fewer duration, this cue is not perceived.

KEY WORDS: dialectal comparison, varieties of Spanish, vowels, temporal cues

RECEPCIÓN: 24/06/2015

ACEPTACIÓN: 25/08/2015 


\section{COMPARACIÓN DIALECTAL DEL ESPAÑOL LIMEÑO Y MADRILEÑO: CONTRASTE ACÚSTICO Y PERCEPTIVO DE LAS VOCALES ${ }^{1}$}

\section{CROSS-DIALECTAL COMPARISON OF LIMA AND MADRID SPANISH: ACOUSTIC AND PERCEPTUAL COMPARISON OF THE VOWELS}

Paloma Pinillos

Pontificia Universidad Católica del Perú

\section{Introducción}

Dentro del campo de la fonología, se han realizado diversos trabajos sobre la cualidad vocálica de sistemas y variedades lingüísticas con la finalidad de evidenciar si este rasgo se encuentra fonologizado. Estos estudios se han llevado a cabo tanto en el ámbito de la producción como en el de la percepción, y han demostrado que existe un contraste a nivel dialectal. En otras palabras, cada sistema lingüístico muestra cualidades acústicas propias, es decir, existe evidencia que indica

${ }^{1}$ La primera versión de este artículo fue presentada como Trabajo de Fin de Máster del Máster de Fonética y Fonología del Posgrado Oficial Estudios Fónicos del CSIC y la UIMP bajo el título de "Producción y percepción de las vocales del español: comparación dialectal entre el español limeño y el español madrileño", y fue dirigido por Mark Aaron Gibson. 
que los hablantes de cada comunidad lingüística computan de forma propia las categorías de sus variedades dialectales.

Según la sociolingüística cognitiva, el entorno en el cual los hablantes se encuentran determina cómo es que llevan a cabo la realización de su variedad. Lo importante es que este ambiente en el que se desarrollan los hablantes y, por tanto, donde se desarrolla también su lengua materna, hace que la percepción de sus sistemas se vea determinada. Respecto a este punto, Moreno señala lo siguiente sobre la fonología cognitiva: "Estos, en tanto los oyentes, ordenan los rasgos percibidos como acústicamente relevantes en categorías fonéticas discretas, primando la información relativa a la identidad categorial y diluyendo la información sobre la forma acústica concreta" (Moreno, 2010: 482). Asimismo, el modelo de adquisición de L1 propuesto por Escudero afirma que "[...] los oyentes maximizan sus probabilidades de entender a los hablantes al realizar decisiones perceptuales que coinciden con su intención del mensaje. Esto lleva a una fuerte dependencia de la producción dada en el ambiente porque un oyente óptimo manifiesta un sonido que coincide con la producción de los sonidos de su ambiente" (2005: 88). ${ }^{2}$ Así, la percepción de los sonidos del habla dependerá del entorno lingüístico, específicamente de las características particulares de este, en el cual se desarrolle el hablante. En otras palabras, el oyente tendrá un input con producciones específicas según su ambiente y, a partir de este, categorizará los sonidos que sean realizados por los hablantes de ese entorno como propios de su L1.

\footnotetext{
2 Traducción propia.
} 
Esta propuesta se apoya con la teoría de la fonología de ejemplares [REFERENCIA], la cual señala que el hablante va construyendo categorías a partir de una referencia modelo o 'ejemplar'. Para que esto se logre, es necesario que los hechos fónicos muestren determinada similitud, y así puedan ser reconocidos o percibidos como una misma categoría. Además, estos hechos fónicos deben darse de manera continua, es decir, con una frecuencia de producción para que sean conocidos e identificables por el hablante. Este último punto es uno de los más importantes, debido a que "los ejemplares fonológicos se construyen a partir de la frecuencia de unos rasgos fónicos" (Moreno, 2010: 483). En este sentido, se habla de categorías fónicas perceptivas, las cuales se pueden entender como un conjunto de estímulos que se agrupan entre sí debido a la similitud de sus propiedades. De esta forma, los hablantes almacenarán representaciones abstractas a partir de una suma de realizaciones específicas y crearán un modelo o ejemplo representativo. Según Geeraerts, desde el punto de vista de la lingüística, lo "ejemplar" o también conocido como "prototípico" tiene cuatro características principales. En primer lugar, las categorías prototípicas no pueden ser definidas tan solo por el significado de una sola agrupación de atributos. En segundo lugar, dentro de las categorías prototípicas existe semejanza o familiaridad a nivel semántico. En tercer lugar, las categorías prototípicas presentan grados, por lo que existen categorías más representativas que otras. Por último, las categorías prototípicas muestran difuminación en los bordes (2006: 148-150). En este sentido, dentro de la fonología, los fonemas vocálicos serían representados como los prototipos. Como señala 
Taylor, "Un sonido vocal será juzgado como / i /, / e / [...] en virtud de su cercanía con el prototipo, donde los límites de cualquiera de las categorías se establecen por la presencia de categorías vecinas. Sus hallazgos también serían consistentes con una teoría del ejemplar, en el que la representación mental de una vocal se compone de huellas de la memoria de casos encontrados previamente" (2010: 54). ${ }^{3}$

De esta forma, la producción y la percepción de la lengua materna del hablante se ven determinadas por el ambiente en el cual este se ha desarrollado. El oyente establecerá categorías discretas a partir de las producciones que se den en su entorno lingüístico. Estas producciones, para ser percibidas por el oyente como relevantes en su lengua materna, deberán ser constantemente realizadas y habrán de tener similitud entre estas, de modo que el oyente pueda distinguirlas y considerarlas como "ejemplos" o "prototipos" propios de su sistema. En el caso específico de la fonología, el hablante almacenará ejemplares fonológicos a raíz de un conjunto de producciones frecuentes y similares que permitirán al hablante construir una categoría ejemplar o representativa.

Uno de los rasgos categóricamente diferenciales en el español es la duración vocálica. Son diversas las variables que pueden influir, como el acento, la estructura silábica, la sonoridad de la consonante postvocálica, el modo de articulación de la consonante postvocálica, la posición de la frase; y, además, se controlaron la velocidad de elocución y el número de sílabas de la palabra (Marín, 1995: 214). En el caso del español peninsular, se señala que existen duracio-

\footnotetext{
3 Traducción propia.
} 
nes intrínsecas en las vocales, siendo la vocal [a] la de mayor duración (69.63 ms.); siguiéndole la [e] (64.95 ms.) y la [o] (64.15 ms.), y, finalmente, la [u] (60.93 ms.) y la [i] (60.66 ms.). De esta forma, se establecería la siguiente gradación de duración vocálica $[\mathrm{a}]>[\mathrm{e}]>[\mathrm{o}]>[\mathrm{u}]>[\mathrm{i}]$ (Marín, 1995: 217), y se da la propuesta de que "[...] en las vocales del español, a una mayor abertura corresponde una mayor duración" (1995: 218).

En cuanto a la comparación dialectal entre el español de Madrid y el español de Lima, hay evidencia de que ambos muestran cualidades acústicas propias en sus sistemas vocálicos. Por un lado, los resultados más relevantes señalan que la frecuencia fundamental de los hablantes madrileños es $8.8 \%$ más baja que la de los limeños y que las vocales de esta variedad de español de España son 33.9\% más cortas que las producidas por los hablantes que manejan la variedad peruana (Morrison y Escudero, 2007: 1508). Asimismo, se han realizado estudios que tienen como base la consideración de que el español tiene un patrón universal, pero que, sin embargo, las variedades dialectales difieren entre sí (Chládková, Escudero y Boersma, 2011). A partir de un estudio específico de la identidad vocálica, es decir, frecuencia fundamental (F0), altura de la lengua (F1), posición de la lengua en un eje horizontal (F2) y duración, se “[...] sugiere que las cinco vocales tienden a ser ligeramente más largas en el español peruano que en el español ibérico [...]" (2011: 422). De esta forma, las características de las vocales producidas por cada grupo dialectal (F0, F1, F2 y duración) difieren. Uno de los datos más importantes es que existe “[...] una interacción entre categoría vocálica y dialecto, la cual 
es significativa para la duración" (2011: 421). ${ }^{4}$ Esto indicaría que los hablantes de español madrileño y español limeño producen las vocales de forma distinta. Además, otro dato que sugieren los resultados es que los hablantes de español limeño tienden a realizar las vocales ligeramente más largas que los madrileños, lo cual apuntaría a que estos últimos hablan más rápido (2011: 422). Incluso, hay estudios que demuestran que las propiedades del sistema fonológico vocálico de un dialecto serán determinantes en el aprendizaje y categorización de los sonidos de una segunda lengua, debido a que se presentan diferencias acústicas en la producción de las vocales nativas (Escudero y Williams, 2012: 1).

\section{Diseño y metodología}

El objetivo de esta investigación es caracterizar y comparar la duración de los sistemas vocálicos del español limeño y del español madrileño. En específico, se asumen dos propuestas: primero, que los limeños y madrileños producen sus vocales basándose en los hechos fónicos de su entorno; $\mathrm{y}$, segundo, que los hablantes de ambos dialectos perciben (distinguen) y registran (identifican) el indicio temporal, es decir, codifican la duración como propia de su sistema fonológico. A partir de estas, se plantea como hipótesis que los limeños producen vocales temporalmente con mayor duración, las discriminan perceptualmente y las registran como propias de su sistema fonológico; mientras que los

\footnotetext{
${ }^{4}$ Traducción propia.
} 
madrileños realizan vocales temporalmente con menor duración, en comparación a las limeñas, las cuales las identifican como parte de su sistema. Se demostraría, entonces, que tanto los limeños como los madrileños establecen determinadas categorías discretas en el nivel cognitivo, a partir de las realizaciones fónicas propias de sus variedades.

Un total de 40 participantes formaron parte de esta investigación: jóvenes universitarios cuyas edades oscilaban entre los 19 y 25 años. Para poder realizar una comparación uniforme de la duración de cada uno de los sistemas vocálicos de las variedades, fue considerada una misma cantidad de hablantes tanto del español madrileño como del limeño: 20 por cada variedad en total. La variable que distingue a ambos grupos es la variedad dialectal española que manejan. Esta es esencial para la comprobación de lo planteado debido a que es una variable dependiente, es decir, determinará los resultados. Sin embargo, también se consideró que los participantes hayan residido durante toda su vida en Madrid o en Lima y que no hayan vivido en otros lugares de habla hispana. Además, se consideró relevante que los padres de los participantes también sean de estas ciudades y manejen la variedad correspondiente, pues, de esa forma, se asegura que los participantes se hayan criado con esta variedad. Estas variables se consideran relevantes, pues permite que los participantes sean controlados, en la medida de lo posible, y, así, resulten grupos más homogéneos para la comparación. Por otro lado, para controlar la variable género, se consideró que cada grupo contara con la misma cantidad de hombres como de mujeres, por lo que cada uno se conformó por 10 de cada sexo. 
Con la finalidad de probar lo propuesto, se diseñaron dos pruebas: una de producción y otra de percepción. La primera consistió en grabar a los 40 participantes produciendo las cinco vocales dentro la estructura ataque-vocalataque-vocal, la cual fue insertada en la frase 'Diga la palabra X, por favor. Esta frase fue leída por cada uno de los participantes. En esta estructura, ambas vocales fueron las mismas, por ejemplo, ataque - $\mathrm{A}$ - ataque - $\mathrm{A}$, ataque $\mathrm{E}$ - ataque - E, etc., y, así, evitar efectos coarticulatorios. En el caso del ataque, la consonante utilizada es la oclusiva bilabial sorda /p/, porque articular este sonido no involucra el desplazamiento y movimiento de la lengua, lo que evita efectos tanto en el primer como en el segundo formante de la vocal. De este modo, los participantes produjeron las frases 'Diga la palabra papa, por favor', 'Diga la palabra pepe, por favor,' 'Diga la palabra pipi, por favor', 'Diga la palabra popo, por favor' y 'Diga la palabra pupu, por favor'. Cada una de estas frases fue repetida 4 veces en un orden aleatorio brindado por el investigador. La finalidad de esta prueba de producción fue corroborar que las vocales del español limeño son más largas que las del español madrileño.

En el caso de la prueba de percepción, se realizó un test de goodness judgement haciendo uso del programa Praat (Boersma y Weenink, 2009). Para esta, se grabó a 2 madrileños y a 2 limeños (los cuales no forman parte de los 20 participantes), 1 hombre y 1 mujer por cada variedad. Se consideró relevante que sean 2 por variedad, un hombre y una mujer, para evitar que los participantes se vean condicionados por el género al escuchar los audios. Estas 4 personas produjeron las vocales en el contexto [oclusiva bilabial 
sorda + vocal], es decir, [pa], [pe], [pi], [po] y [pu]. Se seleccionó la oclusiva bilabial sorda para no alterar las propiedades acústicas de las vocales. La secuencia final que estas personas produjeron fue [pa] [pe] [pi] [po] [pu] [pa], en donde se agrega un [pa] final para evitar efectos acústicos en [pu], como el acortamiento de la duración de la vocal por encontrarse al final de la secuencia. Esta secuencia fue grabada 4 veces en cada uno de los casos para lograr un aproximado más cercano a la duración de las vocales. De esta secuencia, se aislaron las vocales y se obtuvo la media de estas. El resultado de estos promedios base fue el utilizado como estímulo para los participantes de la prueba (cuadro 1$).^{5}$

Cuadro 1. Promedios base de las duraciones de las vocales de hombres y mujeres de Madrid y de Lima utilizadas en esta investigación

\begin{tabular}{|c|c|c|c|c|c|}
\hline \multirow{2}{*}{$\begin{array}{l}\text { Español } \\
\text { Madrid }\end{array}$} & \multicolumn{2}{|c|}{ Duración (ms) } & \multirow{2}{*}{$\begin{array}{c}\text { Español } \\
\text { Lima }\end{array}$} & \multicolumn{2}{|c|}{ Duración (ms) } \\
\hline & Hombre & Mujer & & Hombre & Mujer \\
\hline [a] & 174 & 188 & [a] & 347 & 269 \\
\hline [e] & 180 & 195 & [e] & 318 & 274 \\
\hline [i] & 189 & 187 & [i] & 332 & 288 \\
\hline [o] & 173 & 182 & [o] & 396 & 236 \\
\hline$[\mathrm{u}]$ & 175 & 176 & {$[\mathrm{u}]$} & 293 & 292 \\
\hline
\end{tabular}

\footnotetext{
${ }^{5}$ La diferencia en las duraciones de los sistemas vocálicos de las dos variedades de español es notable. En el caso del español madrileño, la duración de las vocales del hombre oscila entre 173 y 189 ms., siendo la /o/ la vocal más corta y la /i/ la más larga; en cuanto a la mujer, sus duraciones fluctúan entre 176 ms., vocal /u/, y 195 ms., vocal /e/. Por su parte, la variedad limeña presenta duraciones más largas: el hombre registra la duración más corta con la vocal /u/, 293 ms. y la más larga con la /o/, 396 ms.; mientras que la vocal con mayor duración de la mujer es la /i/, 288 ms., y la de menor es la /o/, con 236 ms.
} 
La prueba de percepción consistió en hacer oír a los 40 participantes el audio de una misma vocal 2 veces en una secuencia, una vez producida por un/a madrileño/a y otra por un/a limeño/a. Luego de ser escuchadas, el participante seleccionó la vocal con la que más se identificaba (Figura 1). Por ejemplo, los participantes oyeron la vocal [i] producida tanto por un hombre madrileño y un hombre limeño y tuvieron que seleccionar una de ellas, según con cual se sientan más identificados; y así con cada una de las vocales. Una vez elegida la vocal, el participante seleccionó en la escala del 1 al 5 qué tan seguro estaba de que esta vocal lo identificaba, siendo 1 poco seguro y 5 muy seguro. Este proceso se realizó 4 veces por cada una de las vocales y el participante tuvo la opción de repetir una vez los audios en el caso de desear escucharlos de nuevo. En total, fueron escuchados 400 estímulos por cada participante.

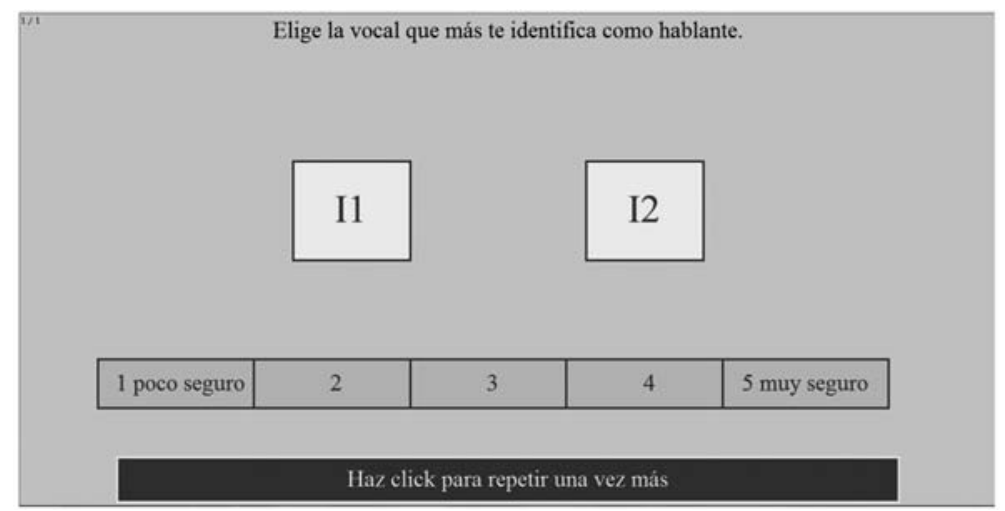

Figura 1. Screenshot de la prueba de percepción en Praat 


\section{Análisis de resultados}

A nivel de producción, la propuesta de esta investigación se comprobó: existen diferencias temporales en la realización de las vocales de los hablantes limeños y madrileños. En otras palabras, se evidencia que las vocales de los limeños tienen mayor duración que las de los madrileños. En el caso de la prueba de percepción, se demostró que los limeños no solo producen vocales con mayor duración, sino también las perciben y categorizan como propias de su sistema fonológico, es decir, el indicio temporal se encuentra representado fonológicamente a nivel perceptual. En cambio, en el caso de los madrileños, a pesar de producir vocales con menor duración, no las registran ni categorizan como parte de su sistema.

Los resultados de la producción de las vocales (cuadro 2) en ambos dialectos reflejaron diferencias significativas. ${ }^{6}$ Por un lado, las mujeres de Lima son las que realizan las vocales con mayor duración en comparación con los hombres de Lima y con los hablantes de Madrid. La vocal [a] de las limeñas es la que registra una duración más extensa: aproximadamente 85.7 ms., lo cual representa un 20.22\% más de duración que la realizada por las madrileñas: $68.37 \mathrm{~ms}$. Además, debe resaltarse que la vocal de mayor duración de las madrileñas es de menor medida que la vocal de menor duración de las limeñas. La vocal de menor duración regis-

\footnotetext{
${ }^{6}$ Para obtener la media de la duración de cada vocal, se obtuvo el promedio de las cuatro vocales producidas por cada uno de los participantes. Las medias de la duración de las vocales de los 40 participantes fueron promediadas para obtener la media total de cada vocal.
} 
Cuadro 2. Comparación de la duración de las vocales del español limeño y madrileño

\begin{tabular}{|c|c|c|c|c|}
\hline \multicolumn{5}{|c|}{ Comparación de la duración vocálica } \\
\hline & Sexo & Madrid & Lima & $\%$ Diferencia \\
\hline \multirow[t]{2}{*}{$\mathrm{a}$} & hombre & 70.85 & 79.05 & 10.37 \\
\hline & mujer & 68.37 & 85.7 & 20.22 \\
\hline \multirow[t]{2}{*}{ e } & hombre & 72.1 & 75.42 & 4.4 \\
\hline & mujer & 69.3 & 76.57 & 9.49 \\
\hline \multirow[t]{2}{*}{$\mathrm{i}$} & hombre & 68.25 & 73.15 & 6.69 \\
\hline & mujer & 66.6 & 80.37 & 17.13 \\
\hline \multirow[t]{2}{*}{ o } & hombre & 68.7 & 73.2 & 6.14 \\
\hline & mujer & 71.75 & 81.85 & 12.33 \\
\hline \multirow[t]{2}{*}{$\mathrm{u}$} & hombre & 65.75 & 72.97 & 9.89 \\
\hline & mujer & 68.3 & 73.3 & 6.82 \\
\hline
\end{tabular}

trada por las mujeres de Lima es la [u] con 73.3 ms.; mientras que las madrileñas obtuvieron la mayor duración con la vocal [o] con $71.75 \mathrm{~ms}$. y la menor duración en [i] con $66.6 \mathrm{~ms}$. De esta forma, el continuo de duración de mayor a menor en el caso de las limeñas sería $[\mathrm{a}]>[\mathrm{o}]>[\mathrm{i}]>[\mathrm{e}]>[\mathrm{u}]$; y el de las madrileñas, $[\mathrm{o}]>[\mathrm{e}]>[\mathrm{a}]>[\mathrm{u}]>[\mathrm{i}]$ (gráfico 1 ). Por otro lado, los hombres de Lima también fueron los que registraron vocales con mayor duración en comparación con los hombres de Madrid. La vocal más extensa producida por los limeños es la [a] con 79.05 ms; la cual supera, en $10.37 \%$, a la realizada por los madrileños (70.85 ms.). Por su parte, los madrileños produjeron con mayor duración la vocal [e] con $72.1 \mathrm{~ms}$. Esta vocal prácticamente coincide con la de menor duración registrada por los limeños: [u] 


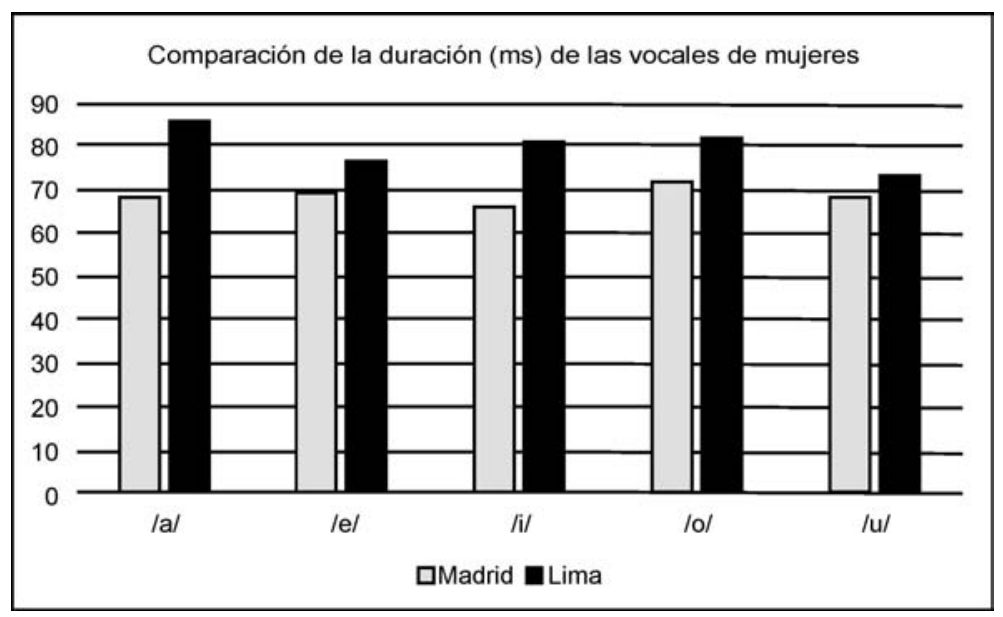

Gráfico 1. Comparación del sistema vocálico de las mujeres madrileñas y limeñas

con $72.97 \mathrm{~ms}$; mientras que la de menor duración de los madrileños presenta una extensión de 65.75 ms. En cuanto a la gradación de mayor de a menor duración, el de los limeños sería $[\mathrm{a}]>[\mathrm{e}]>[\mathrm{o}]>[\mathrm{i}]>[\mathrm{u}]$; y el de los madrileños, $[\mathrm{e}]>[\mathrm{a}]>[\mathrm{o}]>[\mathrm{i}]>[\mathrm{u}]$ (gráfico 2). Además, desde un punto de vista de género, es interesante señalar que si bien las limeñas registran duraciones más extensas que los limeños; en el caso de Madrid, los hombres producen vocales con mayor duración en comparación con las mujeres.

También se realizó una comparación entre los resultados de la investigación de Chládková, Escudero y Boersma (2011), y los obtenidos en esta investigación. Las duraciones obtenidas en ambos trabajos no se alejan mucho. En el caso de los hablantes de Lima (cuadro 3), la duración vocálica de las mujeres es notablemente distinta a la registrada por Chládková et al., sobre todo, en dos de las vocales: [i] 


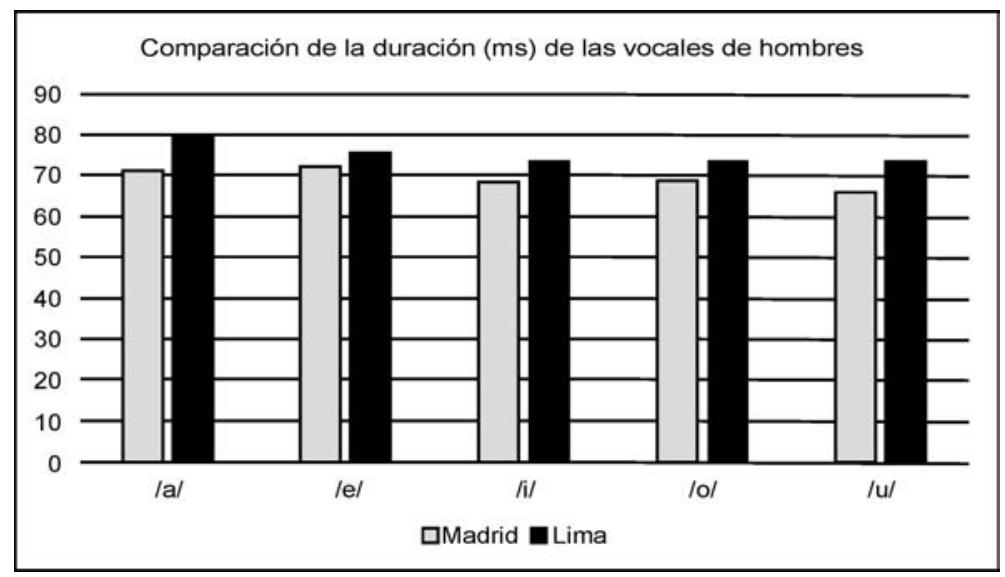

Gráfico 2. Comparación del sistema vocálico de los hombres madrileños y limeños

Cuadro 3. Comparación de la duración de las vocales de español de hablantes limeños de Chládková, Escudero y Boersma (2011), y Pinillos (2014)

\begin{tabular}{|c|c|c|c|c|}
\hline & \multicolumn{2}{|c|}{$\begin{array}{c}\text { Duración de vocales - } \\
\text { mujeres Lima }\end{array}$} & \multicolumn{2}{c|}{$\begin{array}{c}\text { Duración de vocales - } \\
\text { hombres Lima }\end{array}$} \\
\hline Vocal & Chládková et al. & Pinillos & Chládková & Pinillos \\
\hline $\mathrm{a}$ & 87 & 85.7 & 83 & 79.05 \\
\hline $\mathrm{e}$ & 81 & 76.57 & 75 & 75.42 \\
\hline $\mathrm{i}$ & 73 & 80.37 & 67 & 73.15 \\
\hline $\mathrm{o}$ & 81 & 81.85 & 76 & 73.2 \\
\hline $\mathrm{u}$ & 71 & 73.3 & 68 & 72.97 \\
\hline
\end{tabular}

y [e]. El caso con mayor diferencia de duración se da en la vocal [i], ya que estos autores registran una duración de 73 ms.; y en este estudio, 80.37 ms., es decir, 7.37 ms. de diferencia. Por otro lado, la vocal [e] tiene una duración de $81 \mathrm{~ms} .$, mientras que en esta investigación es de $76.57 \mathrm{~ms}$, 
es decir, 4.43 ms. menos. La vocal [o], en cambio, muestra menor contraste, puesto que en esta investigación registró una duración de $81.85 \mathrm{~ms}$. y en la de Chládková et al., 81 ms., en otras palabras, $0.85 \mathrm{~ms}$. de diferencia. En el caso de los hombres, las vocales que más diferencia tienen, en comparación con el estudio de Chládková et al., son la [a] y la [i]. Ese estudio registra una duración de $83 \mathrm{~ms}$. para la vocal [a], en tanto que en este es de 79.05 ms. Por otro lado, en este estudio, la vocal [i] tiene una duración de $73.15 \mathrm{~ms}$., es decir, 6.15 ms. más que en el de Chládková et al., es esta vocal la que muestra un mayor contraste. En el caso de las vocales [o] y [u], en esta investigación, la primera registra una duración de 73.2 ms., mientras que en la otra es de 76 ms; y la segunda, una duración de 72.97 ms., es decir, 4.97 ms. más en comparación con los resultados del otro estudio, $68 \mathrm{~ms}$. Respecto a la vocal [e], el contraste no es significativo, pues solo hay una diferencia de $0.42 \mathrm{~ms}$. entre ambas investigaciones.

En cuanto a los hablantes de Madrid, los resultados obtenidos de la producción vocálica de las mujeres madrileñas también muestran similitudes respecto a la investigación de Chládková et al. (cuadro 4). Sin embargo, hubo un contraste entre la duración de las vocales [a], [e], [o]. La vocal que presenta mayor contraste entre una investigación y otra es la [a], pues hay una diferencia de 16.63 ms., ya que Chládková et al. registra $85 \mathrm{~ms}$., y esta investigación, $68.37 \mathrm{~ms}$. En el caso de la [e], la diferencia también es notable, puesto que en esta investigación se registró 69.3 ms.; mientras que en la otra es de 76 ms., es decir, 6.7 ms. de diferencia. La vocal [o] también presenta diferencia de una investigación a otra. 
Cuadro 4. Comparación de la duración de las vocales del español de hablantes madrileños de Chládková, Escudero y Boersma (2011), y Pinillos (2014)

\begin{tabular}{|c|c|c|c|c|}
\hline & \multicolumn{2}{|c|}{$\begin{array}{c}\text { Duración de vocales - } \\
\text { mujeres Madrid }\end{array}$} & \multicolumn{2}{c|}{$\begin{array}{c}\text { Duración de vocales - } \\
\text { hombres Madrid }\end{array}$} \\
\hline Vocal & Chládková & Pinillos & Chládková & Pinillos \\
\hline $\mathrm{a}$ & 85 & 68.37 & 77 & 70.85 \\
\hline $\mathrm{e}$ & 76 & 69.3 & 69 & 72.1 \\
\hline $\mathrm{i}$ & 69 & 66.6 & 62 & 68.25 \\
\hline $\mathrm{o}$ & 76 & 71.75 & 70 & 68.7 \\
\hline $\mathrm{u}$ & 70 & 68.3 & 66 & 65.75 \\
\hline
\end{tabular}

Chládková et al. registra una duración de 76 ms., mientras que en este estudio la duración es de 71.75 ms., en otras palabras, 4.25 ms. menos. En el caso de las vocales [i] y [u], el contraste es mínimo entre los estudios. Por su parte, en el caso de los hombres, las vocales que muestran mayor diferencia son la [a] y la [i]. En el primer caso, Chládková et al. registra una duración de 77 ms.; mientras que en este estudio la vocal obtuvo un promedio de duración de $70.85 \mathrm{~ms}$., es decir, $6.15 \mathrm{~ms}$. de diferencia. En el caso de la vocal [i], hay una diferencia de 6.25 ms., pues Chládková et al. registra 62 ms.; mientras que esta, $68.25 \mathrm{~ms}$. En el caso de las vocales [e], [o] y [u], la primera resultó con mayor duración que la presentada en Chládková et al. (72.1 ms. frente a $69 \mathrm{~ms}$.); mientras que, en este estudio, la segunda y tercera registraron duraciones menores ( 68.7 frente a 70 ms.; y 65.75 frente a 66). Las diferencias en los resultados obtenidos en ambos estudios pueden ser explicadas si se considera el factor de la heterogeneidad en el habla. A partir de este, se entiende 
que las producciones de los hablantes se caracterizan por compartir rasgos en común, mas no por ser exactamente iguales. Por otro lado, siempre hay factores que son difícilmente controlables y que van a afectar cómo se lleven a cabo las producciones de los hablantes, lo que puede resultar en que dos estudios del mismo fenómeno presenten algunas discrepancias.

Por otro lado, los datos obtenidos en la prueba de percepción también confirman la hipótesis. Los porcentajes de aciertos que obtuvieron los hombres y mujeres según variedad y $\operatorname{sexo}^{7}$ (cuadro 5) presentan diferencias significativas. Los aciertos se interpretan de la siguiente manera: 0 = incorrecto, es decir, la respuesta que dio el hablante no coincide con su variedad dialectal; y 1 = correcto, en otras palabras, la respuesta que dio el hablante coincide con su variedad dialectal. De esta forma, se puede observar que, en el caso de los hablantes de Lima, las mujeres respondieron correctamente en un 80.5\%, e incorrectamente en un 19.5\%; mientras que los hombres acertaron en un $79.2 \%$ y no acertaron en un 20.7\%. En el caso de los hablantes de Madrid, las mujeres respondieron correctamente en un $54.7 \%$ y no coincidieron con su variedad en un $45.2 \%$; por su parte, los hombres acertaron en un 51.2\%, pero fallaron en un $48.7 \%$. En otras palabras, de un total de 400 estímulos, las limeñas acertaron a 322 y los hombres limeños a 317. En cambio, las madrileñas acertaron a 219 y los madrileños a 205 . Todo el grupo de hablantes madrileños tiene un total de 424 respuestas correctas de 800, lo que representa un 53\%. En el

\footnotetext{
${ }^{7}$ Los porcentajes se obtuvieron haciendo uso del programa estadístico SPSS.
} 
Cuadro 5. Tabla de contingencia del porcentaje de aciertos según sexo y variedad

\begin{tabular}{|c|c|c|c|c|c|c|}
\hline \multirow{2}{*}{ Sexo } & & & & \multicolumn{2}{|c|}{ aciertos } & \multirow{2}{*}{ Total } \\
\hline & & & & 0 & 1 & \\
\hline \multirow{4}{*}{ Mujer } & \multirow{4}{*}{ Variedad } & \multirow{2}{*}{ Lima } & \multirow{4}{*}{$\begin{array}{l}\text { Recuento } \\
\% \text { dentro de variedad } \\
\text { Recuento } \\
\% \text { dentro de variedad }\end{array}$} & 78 & 322 & 400 \\
\hline & & & & $19.5 \%$ & $80.5 \%$ & $100.0 \%$ \\
\hline & & \multirow{2}{*}{ Madrid } & & 181 & 219 & 400 \\
\hline & & & & $45.2 \%$ & $54.7 \%$ & $100.0 \%$ \\
\hline \multirow{4}{*}{ Hombre } & \multirow{4}{*}{ Variedad } & \multirow{2}{*}{ Lima } & \multirow{4}{*}{$\begin{array}{l}\text { Recuento } \\
\% \text { dentro de variedad } \\
\text { Recuento } \\
\% \text { dentro de variedad }\end{array}$} & 83 & 317 & 400 \\
\hline & & & & $20.7 \%$ & $79.2 \%$ & $100.0 \%$ \\
\hline & & \multirow{2}{*}{ Madrid } & & 195 & 205 & 400 \\
\hline & & & & $48.7 \%$ & $51.2 \%$ & $100.0 \%$ \\
\hline
\end{tabular}

caso de todo el grupo de limeños, respondió correctamente a 639 de 800 estímulos, es decir, al 79.8\% (cuadro 6). Esto indicaría que este grupo sí tiene tendencia a preferir su variedad dialectal: las vocales con mayor duración. A partir de las cifras de esta muestra, se puede concluir que tanto los hombres como las mujeres madrileñas no tienen tendencia a preferir su variedad, es decir, las vocales con menor duración, sino que les es indiferente este rasgo temporal.

$\mathrm{Al}$ realizarse el análisis de cada una de las vocales por variedad y sexo, se observa que las limeñas acertaron en altos porcentajes casi todas las vocales: entre $83.7 \%$ en /o/ y $92.5 \%$ en /e/, excepto en el caso de la /u/ que registró la menor cantidad de aciertos, 54 correctas de 80, es decir, $67.5 \%$ de aciertos. En el caso de los limeños los porcentajes altos oscilan entre $88.7 \%$ en /a/, y 95\% en /e/, menos la vocal /u/ cuyo valor es de $73,75 \%$, es decir 59 aciertos de 80 (cuadro 7). En el caso de los hablantes de Madrid, las madrileñas tuvieron el mayor porcentaje de acierto $(62.5 \%)$ en la vocal /a/, es decir, 50 correctas y 30 incorrectas; y el menor en /e/ 
Cuadro 6. Tabla de contingencia del número de aciertos por cada grupo de hablantes (Lima y Madrid)

\begin{tabular}{|c|c|c|c|c|c|}
\hline \multirow{2}{*}{ Variedad } & & & \multicolumn{2}{|c|}{ Aciertos } & \multirow{2}{*}{ Total } \\
\hline & & & 0 & 1 & \\
\hline \multirow{3}{*}{ Lima } & & \multirow{3}{*}{$\begin{array}{l}\text { Mujer } \\
\text { Hombre }\end{array}$} & 78 & 322 & 400 \\
\hline & & & 83 & 317 & 400 \\
\hline & \multirow{2}{*}{\multicolumn{2}{|c|}{$\begin{array}{l}\text { Total } \\
\text { Porcentaje }\end{array}$}} & 161 & 639 & 800 \\
\hline \multirow{5}{*}{ Madrid } & & & $20.2 \%$ & $79.8 \%$ & $100 \%$ \\
\hline & \multirow{3}{*}{ Sexo } & Mujer & 181 & 219 & 400 \\
\hline & & Hombre & 195 & 205 & 400 \\
\hline & & & 376 & 424 & 800 \\
\hline & Porc & & $47 \%$ & $53 \%$ & $100 \%$ \\
\hline
\end{tabular}

Cuadro 7. Porcentajes de aciertos y errores de las vocales por variedad y sexo de las mujeres y hombres limeños

\begin{tabular}{|c|c|c|c|c|c|c|}
\hline \multirow{2}{*}{ Variedad } & \multirow{2}{*}{ Sexo } & & \multirow{2}{*}{ Estímulo } & \multicolumn{2}{|c|}{ Aciertos } & \multirow{2}{*}{ Total } \\
\hline & & & & 0 & 1 & \\
\hline \multirow[t]{20}{*}{ Lima } & Mujer & a & Recuento & 11 & 69 & 80 \\
\hline & & & $\%$ & $13.7 \%$ & $86.2 \%$ & $100 \%$ \\
\hline & & $\mathrm{e}$ & Recuento & 6 & 74 & 80 \\
\hline & & & $\%$ & $7.5 \%$ & $92.5 \%$ & $100 \%$ \\
\hline & & $\mathrm{i}$ & Recuento & 10 & 70 & 80 \\
\hline & & & $\%$ & $10.0 \%$ & $90.0 \%$ & $100 \%$ \\
\hline & & o & Recuento & 13 & 67 & 80 \\
\hline & & & $\%$ & $16.2 \%$ & $83.7 \%$ & $100 \%$ \\
\hline & & $\mathrm{u}$ & Recuento & 26 & 54 & 80 \\
\hline & & & $\%$ & $32.5 \%$ & $67.5 \%$ & $100 \%$ \\
\hline & Hombre & $\mathrm{a}$ & Recuento & 9 & 71 & 80 \\
\hline & & & $\%$ & $11.2 \%$ & $88.7 \%$ & $100 \%$ \\
\hline & & e & Recuento & 4 & 76 & 80 \\
\hline & & & $\%$ & $5.0 \%$ & $95.0 \%$ & $100 \%$ \\
\hline & & $\mathrm{i}$ & Recuento & 5 & 75 & 80 \\
\hline & & & $\%$ & $6.2 \%$ & $93.7 \%$ & $100 \%$ \\
\hline & & o & Recuento & 6 & 74 & 80 \\
\hline & & & $\%$ & $7.5 \%$ & $92.5 \%$ & $100 \%$ \\
\hline & & $\mathrm{u}$ & Recuento & 21 & 59 & 80 \\
\hline & & & $\%$ & $26.2 \%$ & $73.75 \%$ & $100 \%$ \\
\hline
\end{tabular}


con 40 aciertos (50\%). Por otra parte, los madrileños obtuvieron $60 \%$ de acierto tanto en /a/ como en /o/ con 48 aciertos, siendo este el porcentaje más alto; mientras que el más bajo es de $53.7 \%$ en la vocal /i/ con un total de 43 correctas (cuadro 8). Un dato importante es que no hay una diferencia significativa entre las respuestas de los hombres y de las mujeres en ambos grupos, pues la relación aciertos - variedad no muestra diferencias según el sexo. De esta forma, en

Cuadro 8. Porcentajes de aciertos y errores de las vocales por variedad y sexo de las mujeres y hombres madrileños

\begin{tabular}{|c|c|c|c|c|c|c|}
\hline \multirow{2}{*}{ Variedad } & \multirow{2}{*}{ Sexo } & & \multirow{2}{*}{ Estímulo } & \multicolumn{2}{|c|}{ Aciertos } & \multirow{2}{*}{ Total } \\
\hline & & & & 0 & 1 & \\
\hline \multirow[t]{20}{*}{ Madrid } & Mujer & a & Recuento & 30 & 50 & 80 \\
\hline & & & $\%$ & $37.5 \%$ & $62.5 \%$ & $100 \%$ \\
\hline & & e & Recuento & 40 & 40 & 80 \\
\hline & & & $\%$ & $50.0 \%$ & $50.0 \%$ & $100 \%$ \\
\hline & & $\mathrm{i}$ & Recuento & 35 & 45 & 80 \\
\hline & & & $\%$ & $43.7 \%$ & $56.2 \%$ & $100 \%$ \\
\hline & & o & Recuento & 39 & 41 & 80 \\
\hline & & & $\%$ & $48.7 \%$ & $51.2 \%$ & $100 \%$ \\
\hline & & $\mathrm{u}$ & Recuento & 37 & 43 & 80 \\
\hline & & & $\%$ & $46.2 \%$ & $53.7 \%$ & $100 \%$ \\
\hline & Hombre & a & Recuento & 32 & 48 & 80 \\
\hline & & & $\%$ & $40.0 \%$ & $60.0 \%$ & $100 \%$ \\
\hline & & e & Recuento & 36 & 44 & 80 \\
\hline & & & $\%$ & $45.0 \%$ & $55.0 \%$ & $100 \%$ \\
\hline & & i & Recuento & 37 & 43 & 80 \\
\hline & & & $\%$ & $46.2 \%$ & $53.7 \%$ & $100 \%$ \\
\hline & & o & Recuento & 32 & 48 & 80 \\
\hline & & & $\%$ & $40.0 \%$ & $60.0 \%$ & $100 \%$ \\
\hline & & $\mathrm{u}$ & Recuento & 36 & 44 & 80 \\
\hline & & & $\%$ & $45.0 \%$ & $55.0 \%$ & $100 \%$ \\
\hline
\end{tabular}


Cuadro 9. Porcentaje de seguridad por vocal de los hablantes limeños

\begin{tabular}{|c|c|c|c|c|c|c|c|c|c|}
\hline \multirow{2}{*}{ Variedad } & \multirow{2}{*}{ Sexo } & \multirow{2}{*}{\multicolumn{2}{|c|}{ Estímulo }} & \multicolumn{5}{|c|}{ Goodness } & \multirow{2}{*}{ Total } \\
\hline & & & & 1 & 2 & 3 & 4 & 5 & \\
\hline \multirow[t]{20}{*}{ Lima } & Mujer & $\mathrm{a}$ & Recuento & & 10 & 4 & 24 & 42 & 80 \\
\hline & & & $\%$ & & $12.5 \%$ & $5.0 \%$ & $30.0 \%$ & $52.5 \%$ & $100 \%$ \\
\hline & & e & Recuento & & 3 & 12 & 20 & 45 & 80 \\
\hline & & & $\%$ & & $3.7 \%$ & $15.0 \%$ & $25.0 \%$ & $56.2 \%$ & $100 \%$ \\
\hline & & i & Recuento & & & 8 & 32 & 40 & 80 \\
\hline & & & $\%$ & & & $10.0 \%$ & $40.0 \%$ & $50.0 \%$ & $100 \%$ \\
\hline & & o & Recuento & & & 10 & 35 & 35 & 80 \\
\hline & & & $\%$ & & & $12.5 \%$ & $43.7 \%$ & $43.7 \%$ & $100 \%$ \\
\hline & & $\mathrm{u}$ & Recuento & 5 & 5 & 12 & 58 & 10 & 80 \\
\hline & & & $\%$ & $6.2 \%$ & $6.2 \%$ & $15.0 \%$ & 72.5 & $12.5 \%$ & $100 \%$ \\
\hline & Hombre & a & Recuento & & & 15 & 35 & 30 & 80 \\
\hline & & & $\%$ & & & $18.7 \%$ & $43.7 \%$ & $37.5 \%$ & $100 \%$ \\
\hline & & e & Recuento & & & 2 & 58 & 40 & 80 \\
\hline & & & $\%$ & & $0.0 \%$ & $2.5 \%$ & $72.5 \%$ & $50.0 \%$ & $100 \%$ \\
\hline & & $\mathrm{i}$ & Recuento & & 24 & 36 & 20 & & 80 \\
\hline & & & $\%$ & & $30.0 \%$ & $45.0 \%$ & $25.0 \%$ & & $100 \%$ \\
\hline & & o & Recuento & & 5 & 12 & 32 & 31 & 80 \\
\hline & & & $\%$ & & $6.2 \%$ & $15.0 \%$ & $40.0 \%$ & $38.7 \%$ & $100 \%$ \\
\hline & & $\mathrm{u}$ & Recuento & & & 5 & 55 & 20 & 80 \\
\hline & & & $\%$ & & & $12.5 \%$ & $68.7 \%$ & $25.0 \%$ & $100 \%$ \\
\hline
\end{tabular}

esta muestra, el sexo no sería una variable que determine la tendencia hacia una variedad $u$ otra, pues arroja porcentajes similares en cada uno de los casos.

Otra de las variables analizadas fue el goodness. Este refleja el grado de seguridad del hablante con respecto a la 
respuesta que dio frente al estímulo escuchado en la prueba. En este caso, se colocó una escala de niveles de seguridad del 1 al 5, en donde 1 es el menor grado de seguridad, y 5 el mayor. Los resultados de goodness por estímulo según variedad y sexo muestran que las mujeres de Lima (cuadro 9) dieron sus respuestas con un nivel de seguridad de 4 y 5 , es decir, alto, con mayor tendencia hacia 5 , en las vocales /a/ (52.5\%), /e/ (56.2\%) e /i/ (50\%). En el caso de la vocal /o/, el grado de seguridad tiende hacia 4 y 5 , y es igual en ambos grados (43.7\%); y en /u/, el grado de seguridad es de 3 y 4 con mayor tendencia hacia 4 (72.5\%). Por su parte, los hombres de Lima muestran un nivel de seguridad de 4 y 5 , con mayor tendencia hacia 4, en el caso de la vocal/a/ (43.7\%). En /e/, el nivel de seguridad con mayor porcentaje es 4 (72.5\%); la vo$\mathrm{cal} / \mathrm{i} /$, por su parte, tiene una mayor seguridad en el grado 3 (45\%); la /o/, en cambio, muestra una seguridad casi igual hacia los grados 4 (40\%) y 5 (38.7\%). Por último, la vocal /u/ registra una seguridad mayor en el grado 4 (68.7\%).

Por su parte, en el resultado de goodness de la variedad de español madrileño (cuadro 10), se puede apreciar que el grado de seguridad de respuesta en las mujeres madrileñas oscila entre 3, 4 y 5. En el caso de las vocales /a/, /e/, /i/, la tendencia de seguridad es hacia el grado 4 con $40 \%$; $56.2 \%$; y $46.2 \%$ respectivamente. En cambio, las vocales /o/ y /u/ muestran mayor seguridad hacia el grado 5 con porcentajes de $52.5 \%$ y $43.7 \%$ correspondientemente. Solo en el caso de la vocal /a/ hay una respuesta, equivalente al 1.2\%, que muestra un grado de seguridad de 1; en el resto de casos, como se mencionó anteriormente, solo hay respuestas entre los grados 3 y 5. Por otro lado, los hombres madrileños tienen una 
Cuadro 10. Porcentaje de seguridad por vocal de los hablantes madrileños

\begin{tabular}{|c|c|c|c|c|c|c|c|c|c|}
\hline \multirow{2}{*}{ Variedad } & \multirow{2}{*}{ Sexo } & \multirow{2}{*}{\multicolumn{2}{|c|}{ Estímulo }} & \multicolumn{5}{|c|}{ Goodness } & \multirow{2}{*}{ Total } \\
\hline & & & & 1 & 2 & 3 & 4 & 5 & \\
\hline \multirow[t]{20}{*}{ Madrid } & Mujer & $\mathrm{a}$ & Recuento & 1 & & 22 & 32 & 25 & 80 \\
\hline & & & $\%$ & $1.2 \%$ & & $27.5 \%$ & $40.0 \%$ & $31.2 \%$ & $100 \%$ \\
\hline & & e & Recuento & & & 16 & 45 & 19 & 80 \\
\hline & & & $\%$ & & & $20.0 \%$ & $56.2 \%$ & $23.7 \%$ & $100 \%$ \\
\hline & & i & Recuento & & & 18 & 37 & 25 & 80 \\
\hline & & & $\%$ & & & $22.5 \%$ & $46.2 \%$ & $31.5 \%$ & $100 \%$ \\
\hline & & o & Recuento & & & 19 & 19 & 42 & 80 \\
\hline & & & $\%$ & & & $23.7 \%$ & $23.7 \%$ & $52.5 \%$ & $100 \%$ \\
\hline & & $\mathrm{u}$ & Recuento & & & 13 & 32 & 35 & 80 \\
\hline & & & $\%$ & & & $16.2 \%$ & $40.0 \%$ & $43.7 \%$ & $100 \%$ \\
\hline & Hombre & $\mathrm{a}$ & Recuento & & & 38 & 22 & 20 & 80 \\
\hline & & & $\%$ & & & $47.5 \%$ & $27.5 \%$ & $25.0 \%$ & $100 \%$ \\
\hline & & e & Recuento & 2 & & 21 & 26 & 31 & 80 \\
\hline & & & $\%$ & $2.5 \%$ & & $26.2 \%$ & $32.5 \%$ & $38.7 \%$ & $100 \%$ \\
\hline & & i & Recuento & & & 20 & 28 & 32 & 80 \\
\hline & & & $\%$ & & & $25.0 \%$ & $35.0 \%$ & $40.0 \%$ & $100 \%$ \\
\hline & & o & Recuento & & & 25 & 25 & 30 & 80 \\
\hline & & & $\%$ & & & $31.2 \%$ & $31.2 \%$ & $37.5 \%$ & $100 \%$ \\
\hline & & $\mathrm{u}$ & Recuento & 1 & 1 & 9 & 32 & 37 & 80 \\
\hline & & & $\%$ & $1.2 \%$ & $1.2 \%$ & $11.2 \%$ & $40.0 \%$ & $46.2 \%$ & $100 \%$ \\
\hline
\end{tabular}

tendencia más o menos proporcional hacia los grados 3,4 y 5 en las vocales /e/, /i/, /o/. En el caso de la vocal/a/, muestra una tendencia de seguridad hacia el grado 3 con un porcentaje de $47.5 \%$. La /u/, en cambio, tiene una tendencia más o menos proporcional hacia los grados 5 y 4 con $46.2 \%$ y $40 \%$ respectivamente. Las únicas dos vocales que presentan 
grados de respuesta de 1 y 2 , pero en porcentajes bajos, son la /e/ y la /u/. En el primer caso, con $2.5 \%$ hacia el grado $1 \mathrm{y}$, en el segundo, 1.2\% tanto hacia el grado 1 como hacia el 2.

\section{Conclusiones}

El presente trabajo cumple con su objetivo central, pues se comprueba que los indicios temporales de los limeños y madrileños son codificados de manera propia, lo cual da evidencia a lo propuesto por Morrison y Escudero (2007) y por Chládková, Escudero y Boersma (2011). De esta forma, a través de la caracterización de los sistemas vocálicos del español hablado en Madrid y en Lima, se prueba que los hablantes de la variedad dialectal limeña producen vocales con mayor duración en comparación con los hablantes de la variedad dialectal madrileña. Esta diferencia en la duración no es exclusiva de un sexo, ya que tanto los hombres como las mujeres de Lima lo realizan. Lo mismo sucede en el caso de los hombres y mujeres de Madrid, debido a que ambos produjeron las vocales con una duración menor a la del grupo de hablantes de Lima.

En cuanto al sexo, en el caso de los hablantes limeños, las mujeres realizan con mayor duración las 5 vocales en comparación con los hombres. La vocal que muestra mayor diferencia en su duración entre ambos sexos es la [o], pues los hombres registran un tiempo de $73.2 \mathrm{~ms}$. y las mujeres de 81.85 ms.; mientras que en la que presentan menor diferencia es la $[\mathrm{u}]$, ya que las mujeres registran $73.3 \mathrm{~ms}$. y los hombres 72.97 ms. Por otro lado, en el caso de los ma- 
drileños, no se presenta un contraste significativo entre las duraciones de los madrileños y madrileñas. Sin embargo, los hombres producen con mayor duración las vocales anteriores [e] (72.1 ms.), [i] (68.25 ms.) y la central [a] (70.85 ms.); mientras que las mujeres registran mayor duración en las posteriores [o] $(71.75 \mathrm{~ms}$.$) y [u] (68.3 \mathrm{~ms}$.).

Además, se presenta mayor contraste entre las duraciones de las mujeres que entre los hombres, es decir, mayor contraste por género. El caso más resaltante en las mujeres se da con la vocal [a], puesto que la de las limeñas registra una duración de 85.7 ms.; mientras que la de las madrileñas es de 68.37 ms., es decir, hay una diferencia de $17.33 \mathrm{~ms}$. En cambio, a pesar de que en el caso de los hombres el mayor contraste también se da en esa vocal, este es menor: $8.2 \mathrm{~ms}$. Asimismo, es relevante resaltar que, en el caso de los hablantes de Lima, la vocal [a] es la producida con mayor duración (79.05 ms. en hombres y 85.7 ms. en mujeres). En el caso de los hablantes de Madrid, los hombres registran la vocal [e] (72.1 ms.) como la de mayor duración, y las mujeres la vocal [o] (71.75 ms.). Sin embargo, en ambas variedades, se presenta un patrón de las vocales con menor registro temporal. Tanto en los hablantes de Lima como en los de Madrid, la vocal $[\mathrm{u}]$ y la vocal [i] tienen una menor duración dentro del continuo vocálico, en otras palabras, las vocales altas.

Por otra parte, en cuanto a los resultados generales de percepción, se comprueba que los hablantes de español limeño distinguen e identifican como propias de su lengua materna las vocales con mayor duración. De esta forma, a partir de esta muestra, se puede proponer que estos hablantes codifican las vocales temporalmente dentro de su re- 
presentación fonológica. En otras palabras, al adquirir su sistema vocálico han establecido determinadas categorías discretas en el nivel cognitivo a partir de las realizaciones fónicas propias de su variedad. Como es sabido, los hablantes producen según lo que perciben y, en el caso de los limeños, se está evidenciando una correspondencia, ya que producen vocales con mayor duración debido a que están expuestos a un entorno con un input de estímulos que presentan estas características. De esta manera, los limeños de esta muestra tendrían fonologizada la duración, es decir, la temporalidad estaría categorizada como un rasgo discreto.

Este resultado apoya la teoría de los ejemplares, ya que se está dando evidencia de que el entorno lingüístico determina qué hechos fónicos el hablante considera como relevantes o ejemplares, a partir de los cuales establece categorías propias de su lengua materna. En otras palabras, el hablante crea categorías o representaciones abstractas de los sonidos a partir de las producciones frecuentes y más prototípicas de su entorno lingüístico. Sin embargo, en el caso de los madrileños la categorización de los indicios temporales de las vocales no se da, puesto que todo indicaría que los hablantes no perciben como propias de su sistema a las vocales con menor duración. A los hablantes de esta variedad les es indiferente la duración de una u otra vocal, es decir, no discriminan como propia una vocal con mayor o menor duración, pues no lo representan como parte de su sistema fonológico. En este caso, se tienen dos posibles opciones: por un lado, que la teoría de correspondencia entre producción y percepción no se estaría cumpliendo, y por otro, que 
los madrileños hayan establecido otro rasgo que no sea de duración que permita identificar sus vocales.

En síntesis, los resultados fueron los esperados: se corroboró lo propuesto por Morrison y Escudero (2007) y Chládková et al. (2011) en cuanto a la realización de las vocales de ambas variedades, por lo que se evidencia que existen diferencias temporales en la producción de las vocales entre limeños y madrileños. Además, se demuestra que los hablantes de la variedad limeña han categorizado los indicios temporales de las vocales de su lengua materna, lo que significa que estas se encuentran representadas fonológicamente a nivel perceptual. Por su parte, el caso de Madrid también resulta interesante, debido a que no se dieron resultados significativos en la prueba de percepción. Sin embargo, si partimos de la misma teoría de ejemplares y de la influencia del entorno acústico, quizá se pueda explicar este caso. Madrid es una ciudad multicultural, en donde muchas hablas son percibidas diariamente. Es probable que un madrileño, a diferencia de un limeño, conviva con un entorno lingüístico más diverso, en donde diferentes registros acústicos del español, e incluso de otras lenguas, forman parte de su input. La heterogeneidad del ambiente ocasionaría que su espectro perceptual sea más amplio, pues estaría conformado por una mayor gama de rasgos acústicos. Como consecuencia, todo indicaría que, en esta muestra, a un madrileño le es indiferente la selección de un rasgo u otro, en este caso, una vocal con mayor o menor duración, pues todo el continuo temporal formaría parte de su espectro. En otras palabras, la gama acústica de temporalidad de un madrileño es tan diversa que no ha identificado ningún rasgo. Por lo 
tanto, su categorización no es significativa, ya que este rasgo se considera irrelevante. Cabe aclarar que esto no significa que se está tomando como presupuesto que el entorno lingüístico de Lima no sea diverso, solo que todo indicaría que el ambiente acústico de Madrid es más heterogéneo en comparación al limeño.

\section{Bibliografía}

Boersma, P. y D. Weenink (2009), "Praat: doing phonetics by computer (version 5.3.84)", en http://www.praat. org/

Chládková, K., P. Escudero y P. Boersma (2011), “Context-specific acoustic differences between Peruvian and Iberian Spanish vowels", Journal of the Acoustical Society of America, 130 (1), pp. 416-428.

Escudero, P. (2005), Linguistic perception and second language acquisition. Explaining the attainment of optimal phonological categorization, tesis doctoral, Utrecht University, LOT Dissertation Series 113.

Escudero, P. y D. Williams (2012), "Native dialect influences second-language vowel perception: Peruvian versus Iberian Spanish learners of Dutch", Journal of the Acoustical Society of America, 131, pp. 406-412.

Geeraerts, D. (2006), "Prospects and problems of prototype theory", en D. Geeraerts y M. De Gruyter (eds.), Cognitive linguistics: basic readings, Deutsche Nationalbibliotek, pp. 141-165. 
MARín, R. (1995), "La duración vocálica en español”, Estudios de Lingüística Universidad de Alicante, 10, pp. 213226.

Moreno Fernández, F. (2010), "Elementos para una fonología cognitiva de la variación", en R. Castañer y V. Lagüéns (eds.), De moneda nunca usada. Estudios filológicos dedicados a José María Enguita, Zaragoza, Institución "Fernando El Católico"-Consejo Superior de Investigaciones Científicas, pp. 471-490.

Morrison, G. y P. Escudero (2007), "A cross-dialect comparison of Peninsula- and Peruvian-Spanish vowels", en International Congress of Phonetic Sciences, Saarbrucken, pp. 1505-1508.

Pinillos, P. (2014), Producción y percepción de las vocales del español: comparación dialectal entre el español limeño y el español madrileño, trabajo de fin de máster, Madrid, Universidad Internacional Menéndez PelayoConsejo Superior de Investigaciones Científicas.

TAYlOR, R. (2010), "Prototypes in Cognitive Linguistics", en P. Robinson y N. Ellis, Handbook of cognitive linguistics and second language acquisition: The Canadian Journal of Linguistics, Nueva York, Routledge, pp. 39-65. 
